\title{
Toxicidade de óleo de mamona a Helicoverpa zea e a Trichogramma pretiosum
}

\author{
Luziani Rezende Bestete ${ }^{(1)}$, Dirceu Pratissoli(2), Vagner Tebaldi de Queiroz ${ }^{(3)}$,
}

Flávio Neves Celestino(2) e Lorena Contarini Machado(2)

(1)Universidade Federal Rural de Pernambuco, Departamento de Agronomia, Avenida Dom Manoel de Medeiros, s/no, Dois Irmãos, CEP 52171-900 Recife, PE. E-mail: Iuziani004@hotmail.com (2)Universidade Federal do Espírito Santo, Centro de Ciências Agrárias, Departamento de Produção Vegetal, Núcleo de Desenvolvimento Científico e Tecnológico em Manejo Fitossanitário, Alto Universitário, s/no, Caixa Postal 16, CEP 29500-000 Alegre, ES. E-mail: pratissoli@cca.ufes.br, fncelestino@hotmail.com, lorenarini@hotmail.com ${ }^{(3)}$ UFES, Departamento de Zootecnia. E-mail: vagnertq@gmail.com

Resumo - O objetivo deste trabalho foi avaliar os efeitos do óleo de mamona sobre a broca-grande do tomateiro, Helicoverpa zea, e sobre o parasitoide de ovos Trichogramma pretiosum. Foi determinada a mortalidade larval da broca, que foi submetida ao óleo de mamona por ingestão e contato às concentrações $0,5,1,0,1,5,2,0,2,5$ e 3,0\% $\left(\mathrm{v} \mathrm{v}^{-1}\right)$. A ação do óleo de mamona sobre T. pretiosum foi determinada mediante testes de seletividade e suscetibilidade. As maiores mortalidades de larvas foram observadas no tratamento por contato com as menores concentrações $(0,5$ e $1,0 \%)$, enquanto com as concentrações intermediárias $(1,5 ; 2,0$ e 2,5\%), não houve diferença entre as vias de aplicação. À maior concentração testada $(3,0 \%)$, o tratamento por ingestão proporcionou mortalidade superior àquela obtida por contato. O número de ovos parasitados por T. pretiosum, no teste de seletividade, foi afetado negativamente pelo óleo de mamona, contudo, os demais parâmetros avaliados quanto à seletividade e à suscetibilidade não foram afetados. O óleo de mamona reduz a sobrevivência de larvas de $H$. zea tanto por ingestão quanto por contato. Além disso, não prejudica o desenvolvimento de T. pretiosum, desde que as pulverizações sejam realizadas após as liberações do parasitoide.

Termos para indexação: Ricinus communis, broca-grande, parasitoide de ovos.

\section{Toxicity of Castor bean oil on Helicoverpa zea and Trichogramma pretiosum}

\begin{abstract}
The objective of this work was to evaluate the effects of castor bean oil on the tomato fruitworm, Helicoverpa zea, and on the egg parasitoid Trichogramma pretiosum. Mortality of tomato fruitworm was determined for larvae subjected to castor bean oil ingestion and contact at concentrations $0.5,1.0,1.5,2.0,2.5 \mathrm{e} 3.0 \%\left(\mathrm{v} \mathrm{v}^{-1}\right)$. The castor bean oil action on T. pretiosum was determined by selectivity and susceptibility tests. The greatest larvae mortalities were observed in the treatment by contact with the lowest concentrations $(0.5$ and $1.0 \%)$, while at intermediate concentrations $(1.5,2.0$, and $2.5 \%)$ there were no differences between the ways of application. At the highest tested concentration of castor bean oil (3.0\%), the ingestion treatment caused a higher mortality, in comparison to the treatment by contact. The number of eggs parasitized by T. pretiosum was negatively affected by castor bean oil in the selectivity test; however, other evaluated parameters for selectivity and susceptibility were not affected. Castor bean oil reduces survival of $H$. zea larvae both by ingestion and contact. In addition, it does not affect the development of $T$. pretiosum, since sprayings are performed after releasing of the parasitoid.
\end{abstract}

Index terms: Ricinus communis, tomato fruitworm, egg parasitoid.

\section{Introdução}

Os danos causados por insetos constituem um dos principais fatores responsáveis por perdas em culturas agrícolas em todo o mundo (Ferry et al., 2004). A broca-grande, Helicoverpa zea (Boddie) (Lepidoptera: Noctuidae), apresenta hábito alimentar polífago e tem sido considerada uma importante praga, provocando consideráveis perdas em diversas culturas, como tomate, algodão e milho (Gould et al., 2002; Lebedenco et al., 2007).
O uso de inseticidas sintéticos tem sido o método mais adotado para o controle de insetos-praga. Para o controle de larvas neonatas de $H$. zea, inseticidas do grupo dos piretroides (Jacobson et al., 2009) têm sido utilizados. No entanto, os diversos problemas associados ao uso extensivo desses inseticidas, como intoxicações e contaminações, têm levado à busca por métodos de controle que ocasionem menor impacto ao ambiente, como o uso de produtos naturais extraídos de plantas (Perez \& Iannacone, 2006). 
Ao longo de sua evolução, determinadas espécies vegetais desenvolveram sua própria defesa química contra insetos herbívoros, ao sintetizar metabólitos secundários com propriedades inseticidas (Wiesbrook, 2004). Entre as várias espécies de plantas com tal propriedade, encontra-se a mamoneira (Ricinus communis L.), cujas sementes contêm substâncias que podem atuar sobre os insetos, impedindo a síntese proteica e inibindo o processo de digestibilidade (Lord et al., 2003; Pantoja-Uceda et al., 2003).

Apesar de não terem sido encontrados dados na literatura quanto à avaliação da atividade inseticida da mamoneira sobre $H$. zea, alguns trabalhos realizados para testar essa atividade, em diferentes espécies de insetos, têm revelado resultados promissores. Ramos-López et al. (2010) mostraram que extratos hexânicos, metanólicos e de acetato de etila de folhas e sementes exerceram atividade inseticida e insetistática em larvas de Spodoptera frugiperda (J.E. Smith) (Lepidoptera: Noctuidae). Igualmente, extratos aquosos e acetônicos de folhas de mamona foram eficientes contra Acromyrmex lundii (Guérin-Méneville) (Hymenoptera: Formicidae) (Caffarini et al., 2008). Resultados satisfatórios também foram encontrados por Mushobozy et al. (2009), que observaram ação inseticida do óleo de mamona em Zabrotes subfasciatus (Boh.) (Coleoptera: Bruchidae).

Outra alternativa à utilização de inseticidas sintéticos para o controle de H. zea, é o uso de parasitoides do gênero Trichogramma West.(Hymenoptera: Trichogrammatidae) - micro-himenópteros que parasitam ovos - e se destacam entre outros inimigos naturais, por sua capacidade de atuar em diversos sistemas agrícolas e florestais e controlar insetos-praga (Hou et al., 2006; Ayvaz et al., 2008). Em meio às várias espécies que podem ser incorporadas ao controle de $H$. zea, linhagens de Trichogramma pretiosum Riley foram relatadas como uma das mais adequadas, por apresentar eficiência de parasitismo superior, em relação a outras espécies avaliadas sobre ovos da praga (Velásquez \& Gerding, 2006). Contudo, o uso de inseticidas associados às liberações desse parasitoide pode interferir no processo de localização e aceitação do hospedeiro e, consequentemente, em sua eficiência (Pinto \& Parra, 2002).

O objetivo deste trabalho foi avaliar os efeitos do óleo de mamona sobre a broca-grande do tomateiro, Helicoverpa zea, e sobre o parasitoide de ovos Trichogramma pretiosum.

\section{Material e Métodos}

O experimento foi realizado no Núcleo de Desenvolvimento Científico e Tecnológico em Manejo Fitossanitário (Nudemafi), do Centro de Ciências Agrárias, da Universidade Federal do Espírito Santo, Alegre, ES.

A criação de $H$. zea foi realizada em sala climatizada $\left[25 \pm 2^{\circ} \mathrm{C}\right.$, umidade relativa do ar (UR) de $70 \pm 10 \%$ e fotofase de 12 horas]. Os adultos foram mantidos em gaiolas de PVC (20 cm de diâmetro x $25 \mathrm{~cm}$ de altura), revestidas internamente com folha de papel ofício branco, com as extremidades fechadas com tecido do tipo "voile"; diariamente, foi oferecida uma solução de mel a $10 \%$ como alimento. Os ovos foram coletados e acondicionados em recipientes de plástico e, após a eclosão, as larvas foram individualizadas em tubos de vidro $(8,5 \times 2,5 \mathrm{~cm})$ que foram preenchidos até $1 / 4 \mathrm{de}$ seu volume com dieta artificial (Greene et al., 1976). As larvas foram mantidas nesses recipientes até o período pupal.

Asfêmeasde T.pretiosum, utilizadas nos experimentos, pertencem à linhagem $T p_{12}$ da coleção do Nudemafi, onde são mantidas e criadas em ovos do hospedeiro alternativo Anagasta kuehniella (Zeller) (Lepidoptera: Pyralidae), conforme Milanez et al. (2009).

Para a obtenção do óleo de mamona, frutos da variedade IAC 80, provenientes de plantações do Município de Muqui, ES, foram coletados e acondicionados em bandejas de plástico $(38 \times 38 \times 9 \mathrm{~cm})$. Posteriormente, foram expostos ao sol para secagem e desprendimento das sementes. As sementes foram submetidas à extração do óleo, mediante processo de prensagem a frio e filtragem das impurezas com filtro de tela fina (malha $50 \mu \mathrm{m}$ ). O óleo foi armazenado em recipiente transparente e fechado hermeticamente, datado e mantido em sala climatizada a $25 \pm 2^{\circ} \mathrm{C}$, com fotofase de 12 horas.

$\mathrm{O}$ experimento foi realizado em câmara climatizada a $25 \pm 1^{\circ} \mathrm{C}$, UR de $70 \pm 10 \%$ e fotofase de 12 horas. A eficiência do óleo de mamona foi avaliada sobre larvas de $H$. zea, submetidas à aplicação por ingestão e contato, às concentrações $0,0,0,5,1,0,1,5,2,0,2,5$ e $3,0 \%\left(\mathrm{v} \mathrm{v}^{-1}\right)$. Para a diluição do óleo, foi utilizada água destilada com o espalhante adesivo Tween 80 a $0,05 \%$ $\left(\mathrm{v} \mathrm{V}^{-1}\right)$ (Isofar Indústria e Comércio de Produtos Químicos Ltda., Duque de Caxias, RJ). A concentração controle de $0,0 \%\left(\mathrm{v} \mathrm{v}^{-1}\right)$ consistiu da aplicação de água destilada com o espalhante adesivo. 
Nos testes de ingestão, foi utilizada uma dieta artificial (Greene et al., 1976) como substrato alimentar. Foram utilizados tubos de acrílico $(10 \mathrm{~mL})$, nos quais a dieta foi depositada a $1 / 3$ do volume total do tubo. Após a solidificação, $50 \mu \mathrm{L}$ da emulsão com óleo de mamona, nas concentrações determinadas, foram aplicados superficialmente à dieta, e os frascos permaneceram abertos durante 60 min em câmara de fluxo laminar. Posteriormente, com o auxílio de um pincel de cerdas finas, larvas de $H$. zea com dois dias de idade foram colocadas individualmente sobre a dieta.

Para avaliar o efeito de contato do óleo de mamona, as larvas foram retiradas da criação e colocadas em placas de Petri descartáveis de $6 \mathrm{~cm}$ de diâmetro revestidas com papel de filtro. Foi realizada a pulverização $(5,5 \mathrm{~mL})$ de cada uma das formulações com óleo de mamona a diferentes concentrações, com o auxílio de torre de Potter (Burkard Scientific, Uxbridge, UK), calibrada para $15 \mathrm{lb} \mathrm{pol}^{-2}$, correspondentes à deposição de $1,78 \mathrm{mg} \mathrm{cm}^{-2}$. As larvas, após receberem as aplicações, foram transferidas para os tubos com dieta.

Os experimentos foram conduzidos em delineamento inteiramente casualizado, em arranjo fatorial $2 \times 7-$ duas vias de aplicação x sete concentrações do óleo de mamona -, com dez repetições, cada uma com cinco larvas, que foram avaliadas diariamente quanto à mortalidade pelo período de dez dias.

A mortalidade por ingestão e contato com óleo de mamona foi corrigida em relação ao tratamento controle pela fórmula de Abbott (1925). Os dados obtidos foram submetidos à análise de variância, e as médias de mortalidade dos tratamentos de ingestão e contato foram comparadas pelo teste t, a $5 \%$ de probabilidade. Para verificar o efeito da concentração do óleo de mamona sobre a mortalidade das larvas, foi realizada uma análise de regressão, a 5\% de probabilidade.

Para a avaliação da seletividade e suscetibilidade de T. pretiosum, foi aplicado o óleo de mamona sobre ovos de H. zea antes (seletividade) e após (suscetibilidade) o parasitismo por T. pretiosum. Foi avaliada a formulação com o óleo $\left(3 \% \mathrm{v} \mathrm{v}^{-1}\right)$ e o espalhante adesivo Tween 80 a $0,05 \%\left(\mathrm{v} \mathrm{v}^{-1}\right)$, pois foi a concentração que causou a maior mortalidade de larvas. Ao tratamento controle, aplicou-se água destilada com o espalhante adesivo.

Em ambos os testes - seletividade e suscetibilidade -, 20 fêmeas, recém-emergidas do parasitoide, foram colocadas individualmente em microtubos tipo Eppendorf $(1,5 \mathrm{~mL})$, que continha uma gotícula de mel em sua parede. Cartelas de cartolina azul celeste
$(2,5 \times 0,5 \mathrm{~cm})$, cobertas com goma arábica diluída a $10 \%$, em que foram colados 15 ovos de H. zea com um dia de idade, foram colocadas em placas de Petri, e os ovos pulverizados $(6,0 \mathrm{~mL})$ com auxílio de torre de Potter $\left(15 \mathrm{lb} \mathrm{pol}^{-2}\right)$. Após a pulverização, as cartelas foram colocadas sobre papel de filtro, à temperatura ambiente, por $60 \mathrm{~min}$, para eliminação do excesso de umidade da superfície dos ovos.

Para o teste de seletividade, cada fềmea inserida nos microtubos recebeu uma cartela com os ovos de $H$. zea pulverizados com o óleo de mamona, para permitir o parasitismo por 24 horas. No teste de suscetibilidade, o parasitismo foi permitido pelo mesmo período e, posteriormente, as cartelas com os ovos já parasitados foram submetidas à pulverização com o óleo de mamona.

Os tratamentos foram mantidos em câmaras climatizadas, reguladas para temperatura de $25 \pm 1^{\circ} \mathrm{C}$, UR de $70 \pm 10 \%$ e fotofase de 14 horas, e observados diariamente durante cinco dias, para eliminação de larvas de $H$. zea recém-eclodidas de ovos não parasitados. Após a emergência, os descendentes foram avaliados no teste de seletividade quanto a: número de ovos parasitados; percentagem de emergência; número de indivíduos por ovo; e razão sexual, calculada por meio do número de fêmeas em relação ao total de indivíduos (machos + fêmeas). No teste de suscetibilidade, foram avaliados os mesmos parâmetros, exceto o número de ovos parasitados.

Empregou-se o delineamento experimental inteiramente casualizado, e os resultados foram submetidos à análise de variância, a 5\% de probabilidade. A redução do parasitismo pelo óleo de mamona foi avaliada pela classificação de toxicidade de produtos fitossanitários, estabelecida pela International Organization for Biological and Integrated Control of Noxious Animals and Plants/West Palearctic Regional Section (IOBC/ WPRS), de acordo com as seguintes categorias: classe 1 , inócuo, toxicidade $<30 \%$; classe 2 , levemente nocivo, toxicidade 30-79\%; classe 3, moderadamente nocivo, toxicidade $80-99 \%$; e classe 4 , nocivo, toxicidade $\geq 99 \%$ (Hassan \& Abdelgader, 2001).

\section{Resultados e Discussão}

A mortalidade larval de $H$. zea variou em consequência da via de aplicação - ingestão ou contato - e da concentração do óleo de mamona, tendo ocorrido interação significativa entre esses fatores $(p=0,0011)$. A via de aplicação por contato ocasionou maior 
mortalidade de larvas nas concentrações 0,5 e 1,0\%, em comparação à aplicação por ingestão (Figura 1). Às concentrações intermediárias - 1,5, 2,0 e 2,5\% -, não houve diferença na mortalidade de larvas entre ingestão e contato. À concentração de $3,0 \%$, houve mortalidade significativamente maior quando as larvas foram tratadas por ingestão.

O óleo de mamona atua sobre $H$. zea tanto por ingestão quanto por contato, a depender da concentração utilizada. Às concentrações de 0,5 e $1,0 \%$, os constituintes químicos da fração óleo ou subprodutos desses compostos, provavelmente, encontram-se em quantidade que permite rápida absorção pelo tegumento das lagartas (Brooks, 1976), suficiente para produzir resposta tóxica superior à da via de aplicação por ingestão. Uma molécula inseticida que chega ao organismo do inseto via ingestão é dependente dos processos de digestão, para incorporação e ação nos sistemas vitais da praga, e o efeito tóxico ocorre em consequência do metabolismo enzimático dos insetos (Dauterman \& Hodgson, 1990; Isman, 2006). Assim, baixas concentrações do óleo de mamona podem ter sido metabolizadas e proporcionado uma manifestação tóxica inferior à da via de aplicação por contato.

A mortalidade de $H$. zea aumentou em razão das concentrações de óleo de mamona via ingestão

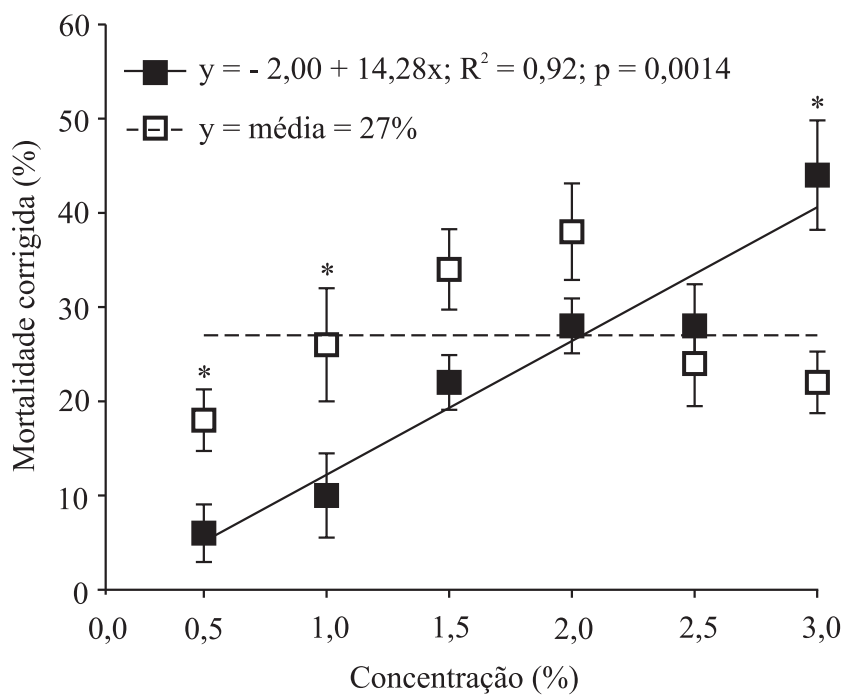

Figura 1. Mortalidade corrigida em relação ao controle (Abbott, 1925) de larvas de Helicoverpa zea, tratadas com óleo de mamona a diferentes concentrações, pelas vias de aplicação ingestão ( $\square$ ) e contato $(\square)$. *Diferença significativa entre as vias de aplicação à mesma concentração, pelo teste t, a $5 \%$ de probabilidade.
(Figura 1). Contudo, os dados de mortalidade obtidos por contato não se ajustaram a nenhum modelo matemático, o que indica que não houve efeito dose-resposta por essa via de aplicação. Sob os tratamentos a concentrações mais elevadas do óleo de mamona, pode ter havido saturação da cera epicuticular do tegumento da lagarta, o que dificultou a penetração do óleo nessas concentrações (Brooks, 1976). Isto justificaria a falta de resposta, pela via de aplicação por contato, a concentrações mais elevadas.

Mortalidade de $44 \%$ de larvas de $H$. zea foi verificada à concentração de $3 \%$, quando o óleo de mamona foi ingerido, e superou os $20 \%$ de mortalidade obtida por contato. Esses resultados assemelham-se aos encontrados por Rondelli et al. (2011), que obtiveram $33,9 \%$ de mortalidade de larvas de Plutella xylostella (L.) (Lepidoptera: Plutellidae), alimentadas com folhas de couve pulverizadas com óleo de mamona à concentração de $2 \%\left(\mathrm{v} \mathrm{v}^{-1}\right)$. Esses mesmos autores verificaram incremento de apenas $10 \%$ na mortalidade larval, quando o óleo foi pulverizado, simultaneamente, sobre folhas e larvas, o que indica menor eficiência do óleo de mamona por contato. A ação de ingestão do óleo de mamona também foi verificada por Ramos-López et al. (2010) que, ao oferecer o óleo de mamona em dieta artificial para larvas de primeiro instar de $S$. frugiperda, à concentração de 16.000 ppm $\left(1,6 \% \mathrm{~V} \mathrm{v}^{-1}\right)$, observaram viabilidade larval nula, tendo sido detectadas atividades insetistáticas do óleo a $560 \mathrm{ppm}\left(0,056 \% \mathrm{v} \mathrm{v}^{-1}\right)$. Santiago et al. (2008) também constataram bioatividade de extratos aquosos do fruto verde de mamoeira a $10 \%$ $\left(\mathrm{v} \mathrm{v}^{-1}\right)$ em $S$. frugiperda, quando adicionados à dieta artificial, o que afetou a duração e a viabilidade larval e a duração e o peso pupal.

A ação inseticida do óleo de mamona sobre lagartas de $H$. zea, pode estar relacionada à presença de alcaloides e proteínas existentes nas sementes da mamona, que agem como inibidores nutricionais para os insetos, uma vez que atuam sobre a $\alpha$-amilase e outras enzimas que degradam polissacarídeos (Pantoja-Uceda et al., 2003).

O óleo de mamona pode ser considerado um produto natural promissor no manejo fitossanitário de $H$. zea, pois pode atuar tanto por ingestão como por contato sobre a praga.

O número de ovos de $H$. zea, parasitados por T. pretiosum no teste de seletividade, foi afetado negativamente pelo óleo de mamona à concentração 
$3 \%$, em comparação ao tratamento controle (Tabela 1). A presença do óleo de mamona na superfície dos ovos de $H$. zea influenciou o comportamento do parasitoide na aceitação do hospedeiro e, consequentemente, reduziu a taxa de parasitismo. O óleo de mamona causou $32,5 \%$ de redução no parasitismo, o que inclui este produto na classe 2 (levemente nocivo, toxicidade $=30-79 \%$ ), pela classificação proposta pela IOBC/WPRS.

Outros produtos naturais já foram relatados como causadores de redução no parasitismo de Trichogramma spp., como observado por Gonçalves-Gervásio \& Vendramim (2004) que, ao utilizar extrato aquoso de sementes de nim a $10 \%\left(\mathrm{v} \mathrm{v}^{-1}\right)$, sobre ovos de A. kuehniella antes do parasitismo de $T$. pretiosum, reduziram drasticamente o número de ovos parasitados, em comparação ao controle. Igualmente, Hohmann et al. (2010) pulverizaram extrato aquoso de sementes de nim a $15 \%\left(\mathrm{v} \mathrm{v}^{-1}\right)$, sobre ovos de A. kuehniella antes do parasitismo de T. pretiosum e Trichogrammatoidea annulata De Santis (Hymenoptera: Trichogrammatiade), e observaram que o número de ovos parasitados foi negativamente afetado. Da mesma forma, Broglio-Micheletti et al. (2006) constataram que o parasitismo por Trichogramma galloi Zucchi (Hymenoptera: Trichogrammatidae), em ovos de Diatraea saccharalis (Fabr.) (Lepidoptera: Crambidae), foi nulo quando os ovos foram tratados com óleo de nim a $0,33,0,53$ e $1 \%\left(\mathrm{v} \mathrm{v}^{-1}\right)$.

Os demais parâmetros avaliados, como percentagem de emergência, número de indivíduos por ovo e razão sexual, tanto no teste de seletividade como no

Tabela 1. Características biológicas e de parasitismo de Trichogramma pretiosum em ovos de Helicoverpa zea tratados com óleo de mamona a $3 \%\left(\mathrm{~V} \mathrm{~V}^{-1}\right)$, à temperatura de $25 \pm 1^{\circ} \mathrm{C}$, umidade relativa $70 \pm 10 \%$ e 14 horas de fotófase ${ }^{(1)}$.

\begin{tabular}{lcccc}
\hline Tratamento & $\begin{array}{c}\text { № de ovos } \\
\text { parasitados }\end{array}$ & $\begin{array}{c}\text { Emergência } \\
(\%)\end{array}$ & $\begin{array}{c}\text { № indivíduos } \\
\text { por ovo }\end{array}$ & $\begin{array}{c}\text { Razão } \\
\text { sexual }\end{array}$ \\
\hline \multicolumn{5}{c}{ Teste de seletividade } \\
Controle & $9,7 \pm 0,40 \mathrm{a}$ & $93,3 \pm 1,54 \mathrm{a}$ & $1,9 \pm 0,06 \mathrm{a}$ & $0,63 \pm 0,02 \mathrm{a}$ \\
Óleo de mamona & $6,5 \pm 0,32 \mathrm{~b}$ & $96,8 \pm 1,25 \mathrm{a}$ & $1,9 \pm 0,10 \mathrm{a}$ & $0,68 \pm 0,05 \mathrm{a}$ \\
\hline \multicolumn{5}{c}{ Teste de suscetibilidade } \\
Controle & $-(2)$ & $79,1 \pm 2,19 \mathrm{a}$ & $1,6 \pm 0,05 \mathrm{a}$ & $0,68 \pm 0,03 \mathrm{a}$ \\
Óleo de mamona & - & $84,6 \pm 3,11 \mathrm{a}$ & $1,7 \pm 0,07 \mathrm{a}$ & $0,68 \pm 0,03 \mathrm{a}$ \\
\hline
\end{tabular}

${ }^{(1)}$ Médias seguidas de letras iguais nas colunas, em cada teste, não diferem pelo teste $\mathrm{F}$, a $5 \%$ de probabilidade. ${ }^{(2)} \mathrm{Ovos}$ tratados após o parasitismo. de suscetibilidade, não foram afetados pelo óleo de mamona, em comparação ao tratamento controle.

A similaridade na percentagem de emergência, obtida em ovos tratados e não tratados, em ambos os testes, indica que a qualidade nutricional desses ovos não foi alterada com a aplicação do óleo de mamona. Esses resultados são semelhantes aos encontrados por Gonçalves-Gervásio \& Vendramim (2004), que observaram que ovos de A. kuehniella tratados antes e após o parasitismo por $T$. pretiosum, com extrato aquoso e clorofórmico de folhas de Trichilia pallida Swartz a $10 \%\left(\mathrm{v} \mathrm{v}^{-1}\right)$, não foram afetados quanto à percentagem de emergência. Porém, esses mesmos autores encontraram diferenças na emergência de T. pretiosum, quando os ovos foram tratados com extrato aquoso de sementes de nim a $10 \%\left(\mathrm{v} \mathrm{v}^{-1}\right)(62,4 \%)$, em comparação ao tratamento controle $(98,6 \%)$.

A ausência de efeitos do óleo de mamona no número médio de indivíduos de $T$. pretiosum, por ovo de H. zea, mostra que o estabelecimento da população do parasitoide não é influenciado pela aplicação do óleo de mamona sobre os ovos, após o processo de parasitismo, na concentração estudada. No caso de aplicações do óleo de mamona antes do parasitismo, apesar de o número de indivíduos por ovo não ter sido afetado, houve redução no parasitismo, o que levaria à redução da população. Navarro \& Marcano (1999) obtiveram resultados inferiores ao do presente trabalho, quanto ao número de indivíduos por ovo, com média de 1,27 indivíduos de $T$. pretiosum por ovo de $H$. zea com um dia de idade. Pratissoli \& Oliveira (1999), ao utilizar o mesmo parasitoide e espécie-praga, encontraram número médio de 1,19 indivíduos por ovo. Entretanto, a densidade de ovos adotada por esses autores diferiu da oferecida ao parasitoide no presente trabalho, o que pode ter contribuído para a diferença de resultados.

Com relação à razão sexual, as taxas encontradas quanto à seletividade e suscetibilidade foram superiores a 0,5 e diferentes de 1 , o que é considerado satisfatório para o controle de pragas. De modo geral, os valores encontrados para os parâmetros biológicos, avaliados em ovos tratados e não tratados, mostram boa adequação dos ovos tratados de H. zea ao desenvolvimento de T. pretiosum.

O óleo de mamona é compatível com a utilização de $T$. pretiosum, desde que as pulverizações sejam realizadas após as liberações do parasitoide, pois, desta forma, esse óleo não influencia a taxa de parasitismo. 


\section{Conclusões}

1. O óleo de mamona, aplicado por contato e por ingestão, aumenta a mortalidade de Helicoverpa zea.

2. O efeito do óleo por contato é maior que por ingestão a concentrações menores que $1,5 \%$.

3. O óleo de mamona a $3 \%$ reduz o número de ovos de H. zea parasitados por Trichogramma pretiosum.

\section{Agradecimentos}

À Coordenação de Aperfeiçoamento de Pessoal de Nível Superior, por concessão da bolsa de estudos; ao Programa de Pós-graduação em Entomologia Agrícola, da Universidade Federal Rural de Pernambuco, e ao Núcleo de Desenvolvimento Científico e Tecnológico em Manejo Fitossanitário, do Centro de Ciências Agrárias, da Universidade Federal do Espírito Santo, pelo apoio técnico e estrutural para o desenvolvimento da pesquisa.

\section{Referências}

ABBOTT, W.S. A method of computing the effectiveness of an insecticide. Journal of Economic Entomology, v.18, p.265-267, 1925.

AYVAZ, A.; KARASU, E.; KARABORKLU, S; YILMAZ, S. Dispersal ability and parasitization performance of egg parasitoid Trichogramma evanescens Westwood (Hymenoptera: Trichogrammatidae) in field and storage conditions. Turkish Journal of Biology, v.32, p.127-133, 2008.

BROGLIO-MICHELETTI, S.M.F.; SANTOS, A.J.N. dos; PEREIRA-BARROS, J.L. Ação de alguns produtos fitossanitários para adultos de Trichogramma galloi Zucchi, 1988 (Hymenoptera: Trichogrammatidae). Ciência eAgrotecnologia, v.30, p.1051-1055, 2006.

BROOKS, G.T. Penetration and distribution of insecticides. In: WILKINSON, C.F. (Ed.). Insecticide biochemistry and physiology. New York: Plenum, 1976. p.3-58.

CAFFARINI, P.; CARRIZO, P.; PELICANO, A.; ROGGERO, P.; PACHECO, J. Efectos de extractos acetónicos y acuosos de Ricinus communis (ricino), Melia azedarach (paraíso) y Trichillia glauca (trichillia), sobre la hormiga negra común. Idesia, v.26, p.59-64, 2008.

DAUTERMAN, W.C.; HODGSON, E. Metabolism of xenobiotics. In: HODGSON, E.; KUHR, R.J. (Ed.). Safer insecticides: development and use. New York: Basel, 1990. p.19-55.

FERRY,N.;EDWARDS,M.G.; GATEHOUSE, J.A.; GATEHOUSE, A.M.R. Plant insect interactions: molecular approaches to insect resistance. Current Opinion in Biotechnology, v.15, p.155-161, 2004.
GONÇALVES-GERVÁSIO, R. deC.R.;VENDRAMIM, J.D. Efeito de extratos de meliáceas sobre o parasitoide de ovos Trichogramma pretiosum Riley (Hymenoptera: Trichogrammatidae). Neotropical Entomology, v.33, p.607-612, 2004.

GOULD, F.; BLAIR, N.; REID, M.; RENNIE, T.L.; LOPEZ, J.; MICINSKI, S. Bacillus thuringiensis-toxin resistance management: stable isotope assessment of alternate host use by Helicoverpa zea. Proceedings of the National Academy of Sciences of the United States of America, v.99, p.16581-16586, 2002.

GREENE, G.L.; LEPPLA, N.C.; DICKERSON, W.A. Velvetbean caterpillar: a rearing procedure and artificial medium. Journal of Economic Entomology, v.69, p.487-488, 1976.

HASSAN, S.A.; ABDELGADER, H. A sequential testing program to assess the side effects of pesticides on Trichogramma cacoeciae Marchal (Hym., Trichogrammatidae). IOBC/WPRS Bulletin, v.24, p.71-81, 2001.

HOHMANN, C.L.; SILVA, F.A.C.; NOVAES, T.G. Selectivity of neem to Trichogramma pretiosum Riley and Trichogrammatoidea annulata De Santis (Hymenoptera: Trichogrammatidae). Neotropical Entomology, v.39, p.985-990, 2010.

HOU, M.L.; WANG, F.L.; WAN, F.G.; ZHANG, F. Parasitism of Helicoverpa assulta Guenée (Lepidoptera: Noctuidae) eggs by Trichogramma spp. (Hymenoptera: Trichogrammatidae): implications for inundative release on tobacco plants. Applied Entomology and Zoology, v.41, p.577-584, 2006.

ISMAN, M.B. Botanical insecticides, deterrents, and repellents in modern agriculture and an increasingly regulated world. Annual Review of Entomology, v.51, p.45-66, 2006.

JACOBSON, A.; FOSTER, R.E.; KRUPKE, C.; HUTCHISON, W.D.; PITTENDRIGH, B.; WEINZIERL, R.A. Resistance to pyrethroid insecticides in Helicoverpa zea (Lepidoptera: Noctuidae) in Indiana and Illinois. Journal of Economic Entomology, v.102, p.2289-2295, 2009.

LEBEDENCO, A.; AUAD, A.M.; KRONKA, S. do N. Métodos de controle de lepidópteros na cultura do tomateiro (Lycopersicon esculentum Mill.). Acta Scientiarum. Agronomy, v.29, p.339-344, 2007.

LORD, M.J.; JOLLIFFE, N.A.; MARSDEN, C.J.; PATEMAN, C.S.; SMITH, D.C.; SPOONER, R.A.; WATSON, P.D.; ROBERTS, L.M. Ricin: mechanisms of cytotoxicity. Toxicological Reviews, v.22, p.53-64, 2003.

MILANEZ, A.M.; PRATISSOLI, D.; POLANCZYK, R.A.; BUENO, A.F. de; TUFIK, C.B.A. Avaliação de Trichogramma spp. para o controle de Trichoplusia ni. Pesquisa Agropecuária Brasileira, v.44, p.1219-1224, 2009.

MUSHOBOZY, D.M.K.; NGANILEVANU, G; RUHEZA, S.; SWELLA, G.B. Plant oils as common bean (Phaseolus vulgaris L.) seed protectants against infestations by the Mexican bean weevil Zabrotes subfasciatus (Boh.). Journal of Plant Protection Research, v.49, p.35-39, 2009.

NAVARRO, R.; MARCANO, R. Preferencia de Trichogramma pretiosum Riley y $T$. atopovirilia Oatman y Platner por huevos de Helicoverpa zea (Boddie) de diferentes edades. Boletín de Entomología Venezolana, v.14, p.87-93, 1999. 
PANTOJA-UCEDA, D.; BRUIX, M.; GIMÉNEZ GALLEGO, G.; RICO, M.; SANTORO, J. Solution structure of RicC3, a S2 albumin storage protein from Ricinus communis. Biochemistry, v.42, p.13839-13847, 2003.

PEREZ, D.D.; IANNACONE, J.O. Efectividad de extractos botánicos de diez plantas sobre la mortalidad y repelencia de larvas de Rhynchophorus palmarum L., insecto plaga del pijuayo Bactris gasipaes Kunth. en la Amazonia del Perú. Agricultura Técnica, v.66, p.21-30, 2006.

PINTO, A.S.; PARRA, J.R.P. Liberação de inimigos naturais. In: PARRA, J.R.P.; BOTELHO, P.S.M.; CORRÊA-FERREIRA, B.S.; BENTO, J.M.S. (Ed.). Controle biológico no Brasil: parasitóides e predadores. São Paulo: Manole, 2002. p.325-342.

PRATISSOLI, D.; DE OLIVEIRA, H.N. de. Influência da idade dos ovos de Helicoverpa zea (Boddie) no parasitismo de Trichogramma pretiosum Riley. Pesquisa Agropecuária Brasileira, v.34, p.891-896, 1999.

RAMOS-LÓPEZ, M.A.; PÉREZ G.S.; RODRÍGUEZ-HERNÁNDEZ, C.; GUEVARA-FEFER, P.; ZAVALA-SÁNCHEZ, M.A. Activity of Ricinus communis (Euphorbiaceae) against Spodoptera frugiperda
(Lepidoptera: Noctuidae). African Journal of Biotechnology, v.9, p.1359-1365, 2010.

RONDELLI, V.M.; PRATISSOLI, D.; POLANCZYK, R.A.; MARQUES, E.J.; STURM, G.M.; TIBURCIO, M.O. Associação do óleo de mamona com Beauveria bassiana no controle da traça-das-crucíferas. Pesquisa Agropecuária Brasileira, v.46, p.212-214, 2011.

SANTIAGO, G.P.; PÁDUA, L.E. de M.; SILVA, P.R.R.; CARVALHO, E.M.S.; MAIA, C.B. Efeitos de extratos de plantas na biologia de Spodoptera frugiperda (J.E. Smith, 1797) (Lepidoptera: Noctuidae) mantida em dieta artificial. Ciência e Agrotecnologia, v.32, p.792-796, 2008.

VELÁSQUEZ, C.F.; GERDING, M.P. Evaluación de diferentes especies de Trichogramma spp. para el control de Helicoverpa zea (Boddie) (Lepidoptera: Noctuidae). Agricultura Técnica, v.66, p.411-415, 2006.

WIESBROOK, M. Natural indeed: are natural insecticides safer and better than conventional insecticides? Illinois Pesticide Review, v.17, p.1-8, 2004.

Recebido em 24 de abril de 2011 e aprovado em 18 de julho de 2011 